\title{
PALEOXIGENACIÓN Y PALEOPRODUCTIVIDAD EN EL GOLFO DE PANAMÁ DURANTE EL HOLOCENO TARDÍO
}

\author{
German D. Patarroyo ${ }^{1}$; José I. Martínez ${ }^{1}$
}

DOI: http://dx.doi.org/10.18273/revbol.v38n2-2016005 (c) (1) Ð

Forma de citar: Patarroyo, G.D., y Martínez, J.I.. 2016. Paleoxigenación y paleoproductividad en el golfo de Panamá durante el Holoceno tardío. Boletín de Geología, 38(2): 75-92.

\section{RESUMEN}

Las condiciones paleoceanográficas del golfo de Panamá para los últimos 4.000 años fueron inferidas a partir del estudio micropaleontológico del núcleo de aguas profundas KNR176-2-MC4 (7.27, $78.24^{\circ} \mathrm{W} ; 2.121$ m.b.n.m.). La relación entre los foraminíferos bentónicos con hábitos infaunales y epifaunales muestra un incremento gradual de las formas infaunales y variaciones en la diversidad hacia el presente, lo que se interpreta como un reflejo de: (1) el detrimento de la oxigenación de los sedimentos de fondo hacia el presente y, (2) mayores efectos tafonómicos que alteran la composición original. Taxa indicadoras de condiciones de moderada oxigenación como Uvigerina proboscidea y Oridorsalis umbonatus fueron encontradas hacia la base del núcleo, mientras que hacia el tope condiciones de mayor productividad superficial y menor oxigenación son inferidas por la presencia común de Globobulimina affinis y U. proboscidea. Otros indicadores de productividad como Epistominella spp. y Uvigerina peregrina son muy comunes hacia la parte media del núcleo.

La comparación del registro micropaleontológico del núcleo MC4 con registros paleoclimáticos del istmo de Panamá (Lago Woodhouse y estalagmita de Chilibrillo) muestra que una mayor recurrencia de eventos tipo El Niño a partir de 5.000 años AP, vendría acompañada de una mayor recurrencia y/o duración de fenómenos de surgencia en el golfo de Panamá. Sin embargo, el registro de susceptibilidad magnética del núcleo MC4 no muestra variaciones significativas que puedan ser comparadas con una disminución en el régimen de precipitación.

Palabras clave: aguas profundas, cuenca de Panamá, foraminíferos bentónicos, Holoceno tardío, paleoceanografía.

\section{LATE HOLOCENE PALEOXYGENATION AND PALEOPRODRUCTIVITY OF THE PANAMA GULF}

\begin{abstract}
Paleocenographic conditions in the Panama Bight for the past 4000 years were inferred by using the micropaleontological record from the deep sea core KNR176-2-MC4 $\left(7.27^{\circ} \mathrm{N}, 78.24^{\circ} \mathrm{W}\right.$; water depth $\left.2121 \mathrm{~m}\right)$. The epifauna-infauna relationship of deep-sea benthic foraminifera and their diversity gradually increase up-core, which is interpreted as a result of: (1) a decrease in the dissolved oxygen content towards the recent and, (2) higher taphonomic effects which modify the original composition of the microfauna. Indicative taxa of moderate oxygen conditions, such as Uvigerina proboscidea and Oridorsalis umbonatus were found at the base of the core. In contrast, lower oxygenation and higher productivity conditions are inferred by the common presence of Globobulimina affinis and Uvigerina proboscidea. Additional paleoproductivity indicators such as Epistominella spp. and Uvigerina peregrina were more common in the middle part of the core.

The comparison of the MC4 core micropaleontological record with several paleoclimatic records from the Panama isthmus (the Woodhouse Lake, and the Chilibrillo stalagmite) illustrates that a higher recurrence of El Niño events since 5000 years BP, could be related to a higher frequency and intensity of upwelling processes in the Panama Gulf. However, the magnetic susceptibility of the MC4 core presents variations which cannot be related with to a reduction in local rainfall.
\end{abstract}

Keywords: deep sea benthic foraminifera, Panama basin, late Holocene, paleoceanography.

${ }^{1}$ Departamento de Geología. Universidad EAFIT, gpatarro@eafit.edu.co,jimartin@eafit.edu.co 


\section{INTRODUCCIÓN}

Los sistemas de surgencia oceánica juegan un papel importante en el ciclo global del carbono debido a las altas tasas de productividad primaria que en estas se generan (e.g. Walsh, 1991). Igualmente, debido a su alta productividad constituyen un renglón importante en la economía representando cerca del $20 \%$ de la producción pesquera a nivel mundial. Esto, teniendo en cuenta que las zonas con surgencia solo ocupan el 1\% del área de los océanos (Pauly and Christensen, 1995).

Estudios globales de las zonas de productividad en los océanos parecen mostrar un incremento en la productividad costera posterior al inicio de la Revolución Industrial y en principio ligado a los fenómenos de calentamiento global (Bakun, 1990; Narayan et al., 2010). Sin embargo, no hay un consenso claro en que estas condiciones de intensificación se hayan presentado en los últimos siglos (Dunbar, 1983; Lemos and Pires, 2004). Por tal motivo, se requieren estudios que vayan más allá en el pasado, siendo los sedimentos de aguas profundas excelentes archivos paleoclimáticos (e.g. Bradley, 1999). Por ejemplo, a través del estudio de las asociaciones de foraminíferos bentónicos de aguas profundas se han encontrado fluctuaciones significativas en las condiciones de productividad para zonas de surgencia en distintas partes del mundo (e.g. Bernhard and Reimers, 1991; Loubere, 1991; Gupta, 1997; Brüchert et al., 2000; Freudenthal et al., 2002; Martins et al., 2006).

Con este enfoque se presentan los resultados del estudio de las asociaciones de foraminíferos bentónicos recuperados del núcleo KNR176-2-MC4 $(38 \mathrm{~cm}$; $7.27^{\circ} \mathrm{N}, 78.24^{\circ} \mathrm{W} ; 2.121$ m.b.n.m), con el propósito de entender la dinámica de surgencia estacional del golfo de Panamá para los últimos miles de años. El conocimiento previo de la ecología de los foraminíferos recientes en esta zona, permite extrapolar sus principales controles biológicos hacia el pasado reciente (Golik and Phleger, 1977). De la misma manera, este estudio busca complementar las observaciones paleoclimáticas efectuadas en continente acerca de la dinámica del fenómeno de El Niño - Oscilación del sur (ENSO) para el Holoceno tardío en Panamá (Bush and Colinvaux, 1994; Lachniet et al., 2004).

\section{AREA DE ESTUDIO}

El golfo de Panamá se encuentra al nororiente de la cuenca de Panamá y se caracteriza por conformar una plataforma carbonatada de gran extensión y poca profundidad (>100 km, $\sim 65$ m; Forsbergh, 1969). Esta plataforma tiene una topografía suave, la cual a los $7-8^{\circ} \mathrm{N}$ da paso a una zona de talud abrupta que supera los $3.000 \mathrm{~m}$ en algunos puntos (Clark, 1988). El núcleo KNR176-2-MC4 $\left(38 \mathrm{~cm} ; 7.27^{\circ} \mathrm{N}, 78.24^{\circ} \mathrm{W} ; 2.121\right.$ m.b.n.m.) fue colectado al suroriente del golfo en la zona batial inferior (FIGURA 1).
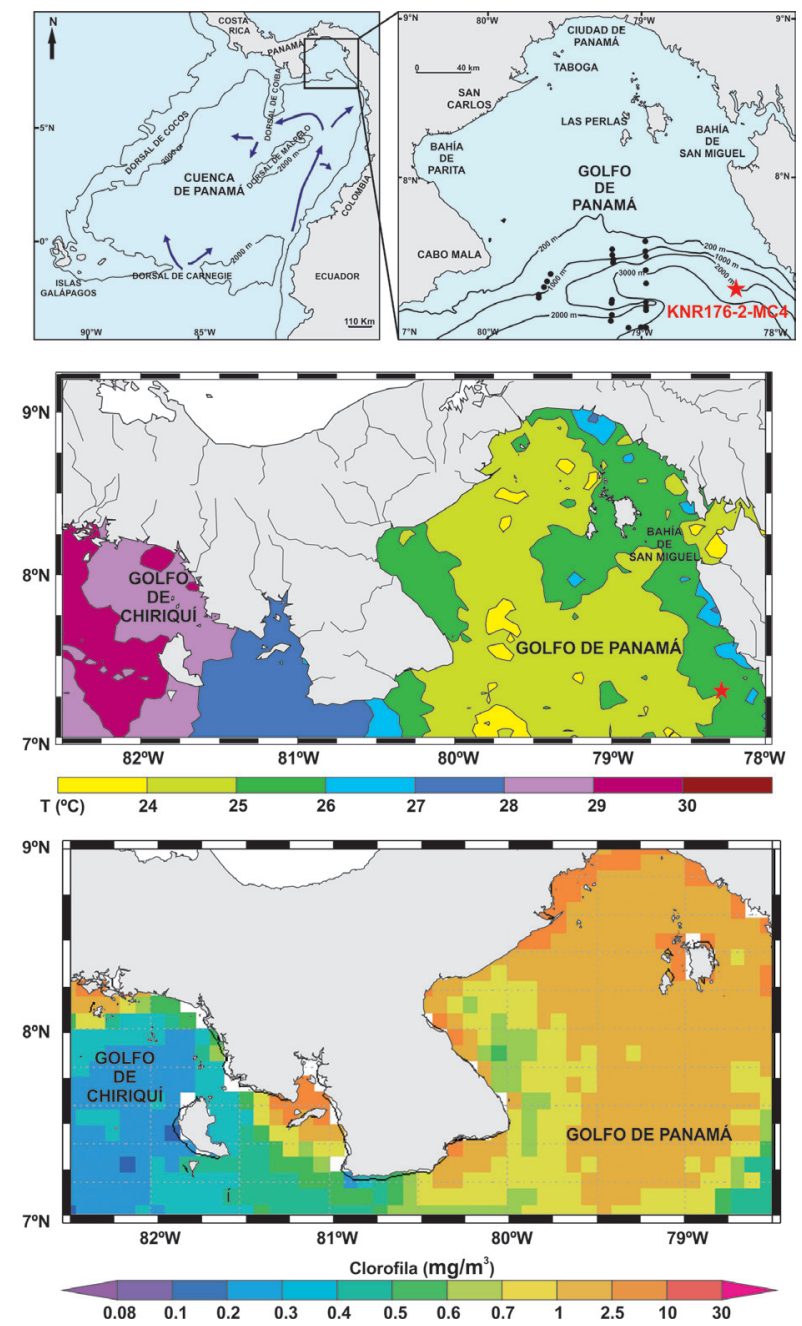

FIGURA 1. Localización del material de estudio. Tope: $(\rightarrow)$ dirección de las corrientes de fondo en la cuenca de Panamá (Lonsdale and Malfait, 1974). En rojo, localización del núcleo KNR176-2-MC4 y en negro las muestras estudiadas por Golik and Phleger (1977). Medio: temperatura superficial del agua en la estación seca (diciembre - marzo) en el golfo de Panamá (modificado de Reijmer et al., 2012). Note la diferencia respecto a las temperaturas del golfo de Chiriquí. Base: Distribución del contenido de clorofila superficial en el golfo de Panamá para la estación seca (modificado de Reijmer et al., 2012). El núcleo KNR176-2-MC4 por extrapolación de los datos estaría ubicado bajo aguas superficiales con contenidos de clorofila entre $0,4-0,6 \mathrm{mg} / \mathrm{m}^{3}$.

La surgencia del golfo de Panamá está condicionada por la intensidad de los vientos alisios del noreste que a su vez responden a la oscilación estacional de la Zona 
de Convergencia Intertropical (ZCIT). Por ende, en el verano austral (diciembre-abril) los fenómenos de surgencia oceánica son particularmente fuertes $\left(45^{*} 10^{4}\right.$ $\mathrm{cm} / \mathrm{s}$ ), al igual que hay un detrimento en los regímenes de precipitación y se genera un sistema atmosférico de pequeña escala denominado el Chorro de Panamá (D’Croz et al., 1991; Chelton et al., 2000; RodríguezRubio et al., 2003; Xie et al., 2005). En contraste, la surgencia oceánica prácticamente desaparece durante el verano boreal (temporada lluviosa) y durante eventos tipo La Niña (Lachniet et al., 2004; Poveda et al., 2006).

La posición promedio de la termoclina se encuentra entre $50-60 \mathrm{~m}$ de profundidad alcanzando los 20 $\mathrm{m}$ durante eventos de surgencia intensos, mientras que la Zona de Oxígeno Mínimo (ZOM) definida por un contenido de oxígeno disuelto $<15 \mu \mathrm{mol}$ $\mathrm{kg}^{-1}$, se encuentra a una profundidad entre 350 y 500 m (FIGURA 2; Fiedler and Talley, 2006; D'Croz and O’Dea, 2007; Reijmer et al., 2012). La posición de la termoclina, más que las variaciones estacionales de la descarga de ríos, constituye la principal fuente de nutrientes en la zona eufótica (e.g. D'Croz and O'Dea, 2007). A su vez, las propiedades fisicoquímicas de las aguas profundas del golfo de Panamá, las cuales son parcialmente alimentadas por las masas de agua provenientes del Pacifico sur, se mantienen relativamente estables a lo largo del año (Fiedler and Talley, 2006).

\section{Oxígeno (ml/l)}

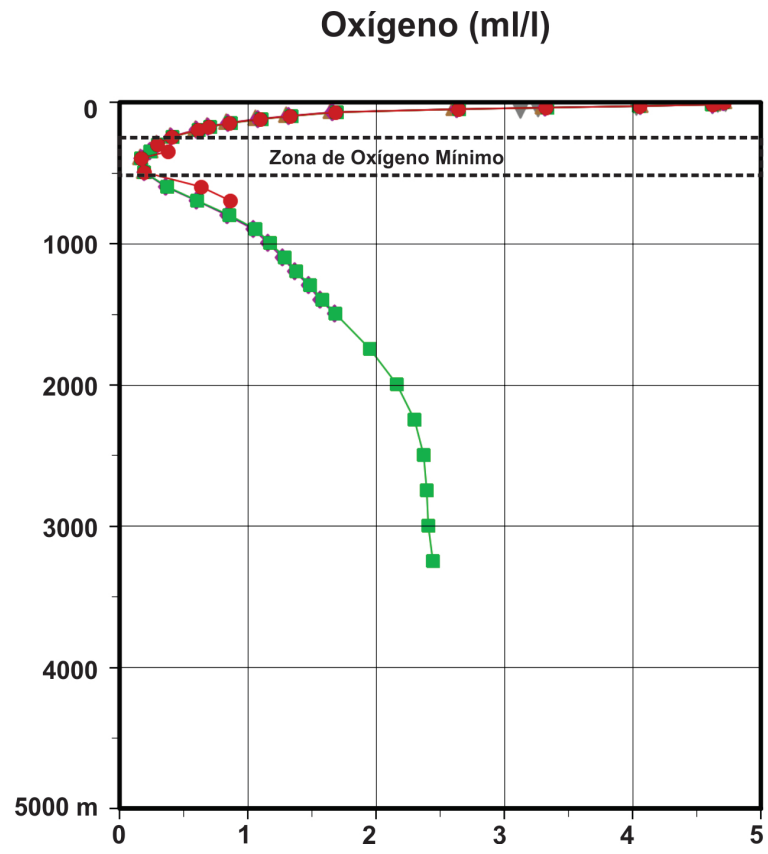

El contenido de carbonato presente en el golfo de Panamá aparentemente no se ve afectado por la surgencia estacional, pero esta si tiene un efecto determinante en la composición biogénica de los sedimentos al interior del golfo. A su vez, la distribución y la producción de carbonato presentan una relación estrecha con el influjo de material terrígeno al golfo, del cual el $45 \%$ proviene de la bahía de San Miguel que es la región con menor productividad superficial (Smayda, 1963; Reijmer et al., 2012). Por último la productividad superficial por los fenómenos de surgencia en el golfo varía entre los 100 y $900 \mathrm{mgC} / \mathrm{m}^{2} / \mathrm{d}$, siendo fácilmente detectada por las concentraciones superiores a $0,6 \mathrm{mg} / \mathrm{m}^{3}$ de la clorofila en el periodo seco (FIGURA 1; Bishop et al., 1986, Reijmer et al., 2012).

Se estima una edad máxima de 4.000 años (Holoceno tardío) para el núcleo KNR176-2-MC4 basado en las tasas de sedimentación calculadas para la cuenca de Panamá y sus zonas circundantes $(10 \mathrm{~cm} / \mathrm{ka}$; Moore et al., 1973; Swift, 1977; Lyle, 1992). La posición distal del núcleo KNR176-2-MC4 respecto al centro del golfo de Panamá, hace poco probable que se haya visto afectado por las variaciones eustáticas reportadas en el Holoceno tardío. Para el centro del golfo de Panamá se estiman variaciones de hasta $50 \mathrm{~m}$ en la columna de agua entre 11.500 y 5.000 años AP (Golik, 1968). Por ende, se sugiere que la paleobatimetría de los sedimentos analizados fue la misma que la batimetría a la que fue colectado el núcleo.

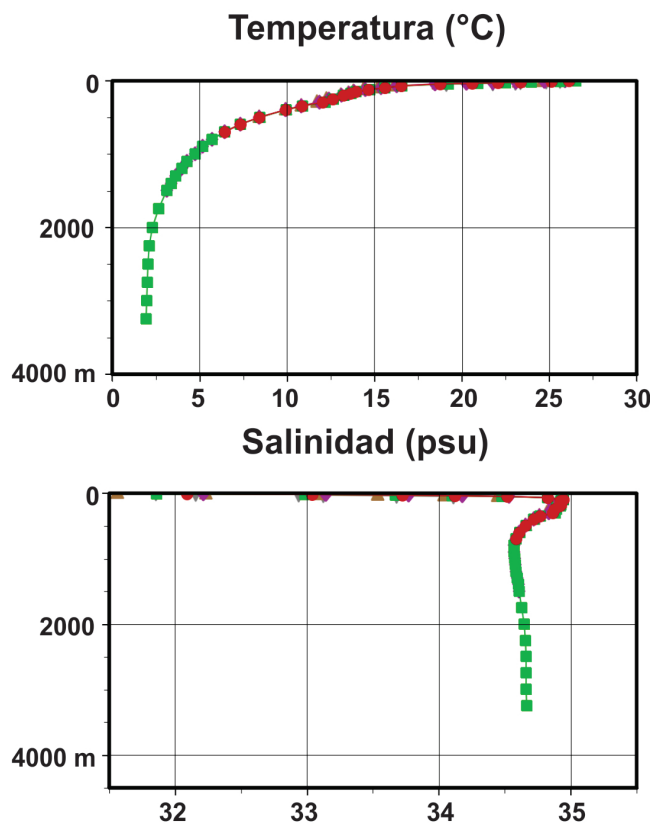

FIGURA 2. Características fisicoquímicas de las aguas del golfo de Panamá (0-3500 m.b.n.m.; estaciones en verde y rojo). Estaciones provenientes del World Ocean Atlas 2009 y graficados con el Ocean Data View ${ }^{\circledR}$. Las estaciones graficadas presentan una ubicación geográfica cercana al núcleo KNR176-2-MC4. 


\section{METODOS}

Se estudiaron un total de 16 muestras, espaciadas cada $2 \mathrm{~cm}$, del núcleo KNR176-2-MC4 (http://dlacruisedata. whoi.edu/KN/KN176L02/index.php) para su contenido de foraminíferos. El material sedimentario $(\sim 5 \mathrm{~g})$ fue procesado y su taxonomía fue determinada siguiendo a: (1) claves taxonómicas (Boltovskoy y Gualancañay, 1975; Clark and Patterson, 1993; Revets, 1996; Loeblich and Tappan, 1964; Bornmalm, 1997; Holbourn and Henderson, 2002; Revets, 2005) y, (2) la información reportada para la cuenca de Panamá (Lalicker and McCulloch, 1940; Cushman and McCulloch, 1942; Bandy and Arnal 1957; Smith, 1963; 1964; Bandy and Rodolfo, 1964; Golik and Phleger, 1977; Gualancañay, 1986; Betancur y Martínez, 2003).

Se estimaron la diversidad y la dominancia de las asociaciones de foraminíferos utilizando los índices de Shannon-Wiener $(\mathrm{H})$ y de equidad ("evenness" E) mediante el Programa Past v. 2.15 (Hammer et al., 2001). El índice de Shannon Wiener indica la relación que se establece entre la cantidad de especies y la abundancia relativa (pi) que ellas presentan en la asociación.

$$
\mathrm{H}=-\Sigma \mathrm{pi} * \ln (\mathrm{pi})
$$

Los valores de este índice parten de 0 , representando este los lugares de menor riqueza y los valores $>0$ los lugares de mayor riqueza de especies (Shannon, 1948). Para el índice de equidad los valores de E siempre son $<1$, presentando sus valores máximos donde las especies (S) son igualmente abundantes en la asociación (Hayek and Buzas, 1997; Magurran, 2004). Este índice ha sido ampliamente usado en estudios de foraminíferos bentónicos (Buzas and Gibson, 1969).

$$
\mathrm{E}=\mathrm{eH} / \mathrm{S}
$$

Igualmente, se hizo énfasis en las proporciones de especies indicadoras de paleoproductividad y paleoxigenación de acuerdo a la literatura (e.g. Rathburn and Corliss, 1994; Bernhard and Sen Gupta, 1999; Jorissen et al., 2007; De and Gupta, 2010).

Estudios de susceptibilidad magnética (tipo másica) fueron efectuados en 38 muestras del núcleo (intervalos de cada $1 \mathrm{~cm}$ ) utilizando $8 \mathrm{~g}$ de material libre de humedad. Para efectuar dichas mediciones $\left(\mathrm{m}^{3} / \mathrm{kg}\right)$ se utilizó el equipo kappabridge MFK1-FB (AGICO) del Laboratorio de Magnetometría de la Universidad EAFIT.

\section{RESULTADOS}

\section{Sedimentología y datos de susceptibilidad magnética}

El núcleo MC4 está constituido por lodolitas de color marrón claro a verdoso y composición mixta de material terrígeno y biogénico. La capa oxidada superficial es de color marrón rojizo y alcanza los $5 \mathrm{~cm}$ de espesor. El registro de susceptibilidad magnética presenta valores que oscilan entre 1,48 y $1,70^{*-6}$ $\mathrm{kg} / \mathrm{m}^{3}$, algo más elevados hacia la parte media (FIGURA 3, TABLA 1), lo que sugiere que no hay variaciones significativas en el contenido de minerales magnéticos (e.g. máficos).

Tabla 1. Valores de susceptibilidad magnética (tipo másico) del núcleo KNR176-2-MC4.

\begin{tabular}{cccc}
\hline Muestra (cm) & $\begin{array}{c}\text { Susceptibilidad } \\
\text { magnética (m } \mathbf{3} / \mathbf{k g})\end{array}$ & Fase & Rango \\
\hline $5-6$ & $1,5228 \mathrm{E}-06$ & 2,00 & $3 / 3$ \\
$6-7$ & $1,6246 \mathrm{E}-06$ & 1,99 & $3 / 3$ \\
$7-8$ & $1,4832 \mathrm{E}-06$ & 1,99 & $3 / 3$ \\
$8-9$ & $1,5043 \mathrm{E}-06$ & 1,98 & $3 / 3$ \\
$9-10$ & $1,5267 \mathrm{E}-06$ & 1,94 & $3 / 3$ \\
$10-11$ & $1,5394 \mathrm{E}-06$ & 1,92 & $3 / 3$ \\
$11-12$ & $1,5339 \mathrm{E}-06$ & 1,92 & $3 / 3$ \\
$12-13$ & $1,5608 \mathrm{E}-06$ & 1,92 & $3 / 3$ \\
$13-14$ & $1,5944 \mathrm{E}-06$ & 1,89 & $3 / 3$ \\
$14-15$ & $1,5940 \mathrm{E}-06$ & 1,90 & $3 / 3$ \\
$15-16$ & $1,6603 \mathrm{E}-06$ & 1,84 & $3 / 3$ \\
$16-17$ & $1,7128 \mathrm{E}-06$ & 1,90 & $3 / 3$ \\
$17-18$ & $1,7306 \mathrm{E}-06$ & 1,85 & $3 / 3$ \\
$18-19$ & $1,7593 \mathrm{E}-06$ & 1,86 & $3 / 3$ \\
$19-20$ & $1,7931 \mathrm{E}-06$ & 1,85 & $3 / 3$ \\
$20-21$ & $1,6484 \mathrm{E}-06$ & 1,84 & $3 / 3$ \\
$21-22$ & $1,6700 \mathrm{E}-06$ & 1,86 & $3 / 3$ \\
$22-23$ & $1,6649 \mathrm{E}-06$ & 1,88 & $3 / 3$ \\
$23-24$ & $1,6124 \mathrm{E}-06$ & 1,86 & $3 / 3$ \\
$24-25$ & $1,6910 \mathrm{E}-06$ & 1,84 & $3 / 3$ \\
$25-26$ & $1,7488 \mathrm{E}-06$ & 1,82 & $3 / 3$ \\
$26-27$ & $1,6837 \mathrm{E}-06$ & 1,86 & $3 / 3$ \\
$27-28$ & $1,6947 \mathrm{E}-06$ & 1,85 & $3 / 3$ \\
$28-29$ & $1,7577 \mathrm{E}-06$ & 1,84 & $3 / 3$ \\
$29-30$ & $1,6846 \mathrm{E}-06$ & 1,85 & $3 / 3$ \\
$30-31$ & $1,6145 \mathrm{E}-06$ & 1,85 & $3 / 3$ \\
$31-32$ & $1,5571 \mathrm{E}-06$ & 1,84 & $3 / 3$ \\
$32-33$ & $1,5138 \mathrm{E}-06$ & 1,89 & $3 / 3$ \\
$33-34$ & $1,4873 \mathrm{E}-06$ & 1,88 & $3 / 3$ \\
$34-35$ & $1,5262 \mathrm{E}-06$ & 1,89 & $3 / 3$ \\
$35-36$ & $1,5581 \mathrm{E}-06$ & 1,81 & $3 / 3$ \\
$36-37$ & $1,5091 \mathrm{E}-06$ & 1,84 & $3 / 3$ \\
$37-38$ & $1,4764 \mathrm{E}-06$ & 1,87 & $3 / 3$ \\
\hline & & & \\
& & & \\
& & &
\end{tabular}


El componente biogénico del núcleo en la fracción $>125 \mu \mathrm{m}$ corresponde, en orden de abundancia, a conchillas de foraminíferos planctónicos y bentónicos, radiolarios, valvas de ostrácodos, micro-moluscos y restos de peces.

\section{Asociaciones de foraminíferos bentónicos}

A pesar de que la composición de la microfauna varía a lo largo del núcleo, se observan algunas tendencias generales en el mismo. El listado de especies principales es referido en el Anexo. Para efectos de visualización se describe el núcleo en dos intervalos:

Intervalo 40-30 cm ( 3-4 ka AP): En general, las conchillas de foraminíferos de tipo aglutinado presentan un incremento leve hacia la base mientras que las conchillas de tipo hialino y porcelanáceo no presentan un patrón determinado con la profundidad (FIGURA 3; TABLA 2). Las formas aglutinantes más comunes corresponden a especies de los géneros Lagenammina, Eggerella, Paratrochammina, Saccammina, Textularia y Reophax. Las asociaciones de foraminíferos presentan diversidades bajas en la base $(\mathrm{H}<3.5)$ mientras que el índice de equidad $(\mathrm{E}>0,6)$ evidencia la dominancia baja de especies particulares en el intervalo 33-35 cm (FIGURA 3; TABLA 2). Las asociaciones de foraminíferos hialinos se encuentran dominadas por distintos géneros de las familias Bolivinidae, Uvigerinidae y Buliminidae. El género Bolivina alcanza gran representatividad con las especies B. humilis, B. pacifica, B. seminuda у B. semiperforata, $\mathrm{y}$ en menor medida por $B$. subadvena y B. plicata, siendo sus abundancias relativamente constantes desde la base del núcleo. La familia Uvigerinidae se encuentra representada por distintas especies de los géneros Uvigerina y Trifarina, de las cuales se destaca el predominio de U. proboscidea y U. hispida hacia la base (FIGURA 4). A su vez, la familia Buliminadae se encuentra representada por los géneros Bulimina, Globobulimina y Buliminella pero en una proporción más baja que las otras familias descritas. Otras especies con presencia significativa corresponden a Epistominella spp., Cibicidoides bradyi y Cassidulina carinata las cuales difieren en sus proporciones con la profundidad. Para el caso de Epistominella (E. exigua y E. smithi) sus mayores abundancias se encuentran hacia la parte media-baja del núcleo.
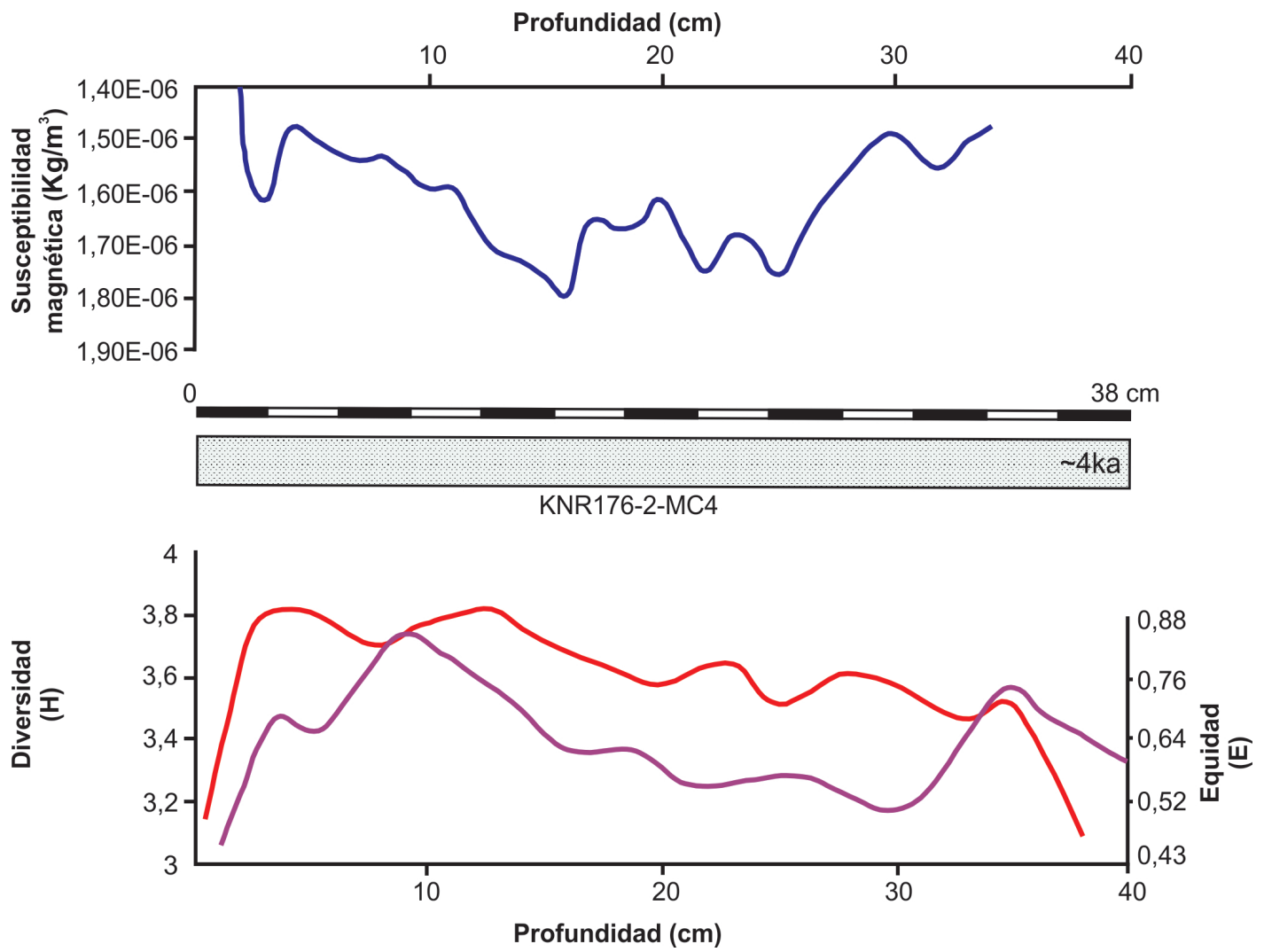

FIGURA 3. Valores de susceptibilidad magnético (tipo másico) y tendencias de los índices de diversidad (rojo) y equidad (morado) de las asociaciones de foraminíferos bentónicos del núcleo KNR176-2-MC4. Nótense los valores moderadamente superiores de la susceptibilidad magnética hacia la parte media del núcleo, y el incremento en los valores de $\mathrm{H}$ y E hacia el tope del núcleo. 
Tabla 2. Valores porcentuales de los foraminíferos bentónicos según el tipo de conchilla, y valores de diversidad y equidad del núcleo KNR176-2-MC4.

\begin{tabular}{cccccc}
\hline & & & & \\
\hline 1 & 58,49 & 0 & 41,51 & 3,16 & 0,44 \\
3 & 4,79 & 0 & 95,21 & 3,76 & 0,63 \\
5 & 2,86 & 0,63 & 96,51 & 3,81 & 0,62 \\
8 & 18,93 & 6,05 & 75,03 & 3,70 & 0,77 \\
10 & 4,07 & 5,69 & 90,24 & 3,76 & 0,74 \\
13 & 6,41 & 1,42 & 92,17 & 3,82 & 0,66 \\
15 & 2,61 & 1,40 & 96,00 & 3,73 & 0,59 \\
18 & 2,34 & 0,66 & 97,00 & 3,63 & 0,58 \\
20 & 0,89 & 0,99 & 98,13 & 3,57 & 0,53 \\
23 & 1,93 & 0,64 & 97,43 & 3,65 & 0,54 \\
25 & 2,23 & 3,75 & 94,06 & 3,51 & 0,54 \\
28 & 5,21 & 2,27 & 92,56 & 3,62 & 0,49 \\
30 & 1,22 & 2,92 & 95,86 & 3,58 & 0,53 \\
33 & 4,03 & 5,65 & 90,32 & 3,47 & 0,69 \\
35 & 9,29 & 2,14 & 88,57 & 3,52 & 0,64 \\
38 & 10,70 & 4,95 & 84,35 & 3,10 & 0,57 \\
\hline
\end{tabular}

Intervalo 30-0 cm (reciente-3 ka AP): Las formas aglutinadas decrecen hacia la parte media y tienden a ser predominantes $(>10 \%)$ en los últimos $5 \mathrm{~cm}$ del núcleo. En contraste las conchillas de tipo hialino y porcelanáceo no presentan un patrón determinado con la profundidad (FIGURA 3; TABLA 2). Entre las formas porcelanáceas más comunes se encuentran Pyrgo oblonga, Quinqueloculina venusta y Triloculina tricarinata. La diversidad de las asociaciones tiende a ser mayor $(\mathrm{H}>3.7)$ entre los 7 y $12 \mathrm{~cm}$, mientras que el índice de equidad $(\mathrm{E}>0,6)$ evidencia la dominancia baja de especies hacia los 8-10 cm (FIGURA 3; TABLA 2). Las asociaciones de foraminíferos hialinos se encuentran dominadas por distintos géneros de las familias Bolivinidae, Uvigerinidae y Buliminidae. De estos, el género Brizalina (B. argentea, $B$. interjuncta) tiende a aumentar hacia el tope del núcleo y las especies Trifarina bradyi y Uvigerina peregrina son comunes hacia la parte media del núcleo (FIGURA 4). A su vez, el género Globobulimina ( $G$. auriculata y $G$. affinis) es abundante entre los 7 y $10 \mathrm{~cm}$, precedido de un aumento relativo en la proporción del género Bulimina hacia la parte más superior del núcleo $(0-5 \mathrm{~cm})$.

Otras especies como Cassidulina carinata y Cibicidoides bradyi tienden a ser más comunes hacia la parte superior del núcleo. Por último, algunas especies con proporciones menos significativas $(<7 \%)$ presentan diversas tendencias con la profundidad. Tal es el caso de Melonis spp. que presenta un incremento leve hacia los $20 \mathrm{~cm}$, Oridorsalis umbonatus cuya proporción disminuye por debajo del nivel de oxidación (5-8 cm), o la aparición de Fursenkoina spp. que es intermitente (FIGURA 4).

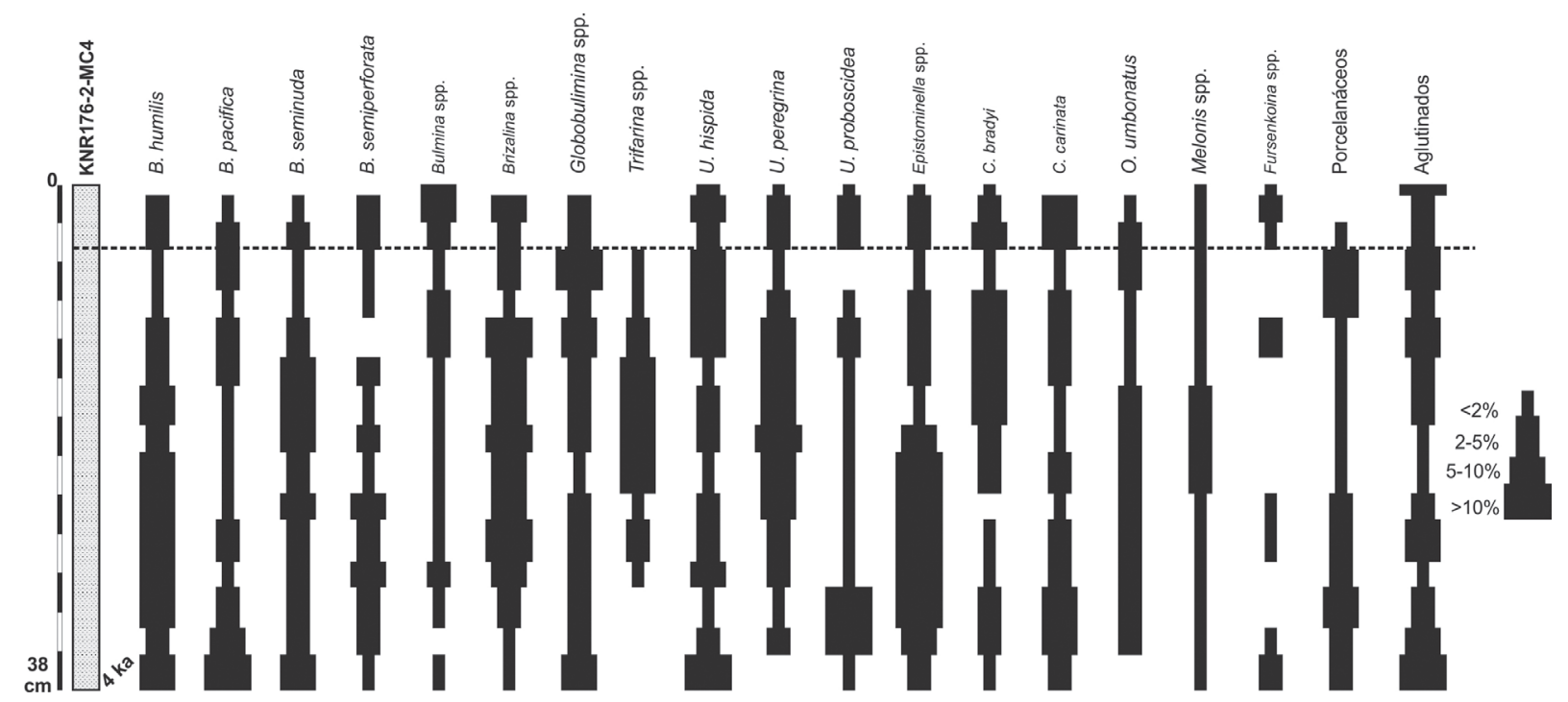

FIGURA 4. Distribución porcentual de las principales especies y grupos de foraminíferos bentónicos en el núcleo KNR176-2MC4. La línea punteada indica la profundidad de la capa oxidada.

Viendo al núcleo como un conjunto nuevemante, se observó que especies comunes en sedimentos de aguas profundas como Globocassidulina subglobosa, Cibicidoides wuellerstorfi, Hoeglundina elegans,
Laticarinina pauperata, Valvulineria humilis, Loxostomum pseudobeyrichi, Suggrunda eckisi y Epistominella pacifica están presentes en proporciones bajas y no muestran tendencias particulares a lo largo del núcleo. 


\section{DISCUSIÓN}

En vista de los patrones de distribución de las asociaciones de foraminíferos en el núcleo MC4 es evidente que tres factores serían determinantes: (1) variaciones en las condiciones paleoecológicas en los últimos miles de años, (2) procesos tafonómicos y, (3) las preferencias ambientales de las especies encontradas.

Por ejemplo, para la parte más basal del núcleo (Intervalo $30-40 \mathrm{~cm}$ ), la gran proporción de la especie infaunal $U$. proboscidea, así como la abundancia baja de taxa como Bolivina, Brizalina y C. bradyi, sugieren condiciones de oxigenación moderada en los sedimentos (e.g. Gupta, 1997; Bornmalm et al., 1999). Sin embargo, a partir de los $38 \mathrm{~cm}$ hay un cambio en las asociaciones de foraminíferos que se caracteriza por un aumento en la proporción de Bolivina, Brizalina y C. bradyi, y la aparición común a moderada de especies como U. peregrina, Trifarina spp. y O. umbonatus (FIGURA4). La presencia de estas especies junto con la proporción constante de Epistominella spp. sugieren condiciones de alta productividad superficial y baja oxigenación en los sedimentos de fondo (TABLA 3; Loubere, 1999; Fontanier et al., 2002; Licari et al., 2005). Este cambio faunístico también se ve reflejado en los índices de diversidad y equidad (FIGURA 3).

Tabla 3. Valores porcentuales de los foraminíferos epifaunales e infaunales, y de las taxa indicadores de paleoproductividad Epistominella spp. y Uvigerina peregrina para el núcleo KNR176-2-MC4.

\begin{tabular}{|c|c|c|c|c|}
\hline 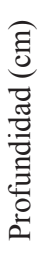 & 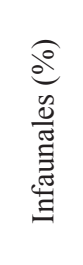 & 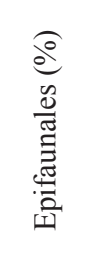 & 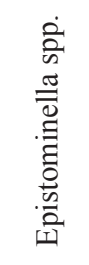 & 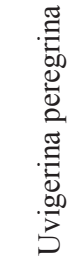 \\
\hline 1 & 61,22 & 38,78 & 0,69 & 0,69 \\
\hline 3 & 81,68 & 19,32 & 4,17 & 3,51 \\
\hline 5 & 74,29 & 25,71 & 4,44 & 3,81 \\
\hline 8 & 76,46 & 23,54 & 0,86 & 0,86 \\
\hline 10 & 73,98 & 26,02 & 4,88 & 4,07 \\
\hline 13 & 79,65 & 21,35 & 2,30 & 7,37 \\
\hline 15 & 73,91 & 26,09 & 4,05 & 8,72 \\
\hline 18 & 73,98 & 26,02 & 1,63 & 9,12 \\
\hline 20 & 77,91 & 22,09 & 7,62 & 12,61 \\
\hline 23 & 70,10 & 29,9 & 12,22 & 8,68 \\
\hline 25 & 70,56 & 29,44 & 14,66 & 7,17 \\
\hline 28 & 75,25 & 24,75 & 10,68 & 4,21 \\
\hline 30 & 69,03 & 31,97 & 13,11 & 4,57 \\
\hline 33 & 68,88 & 31,12 & 16,13 & 1,00 \\
\hline 35 & 72,46 & 28,54 & 9,29 & 3,57 \\
\hline 38 & 83,54 & 17,46 & 2,87 & 0,00 \\
\hline
\end{tabular}

Por ende, asumiendo una tasa constante de sedimentación de 10 cm/ka (sensu Lyle, 1992) para el núcleo MC4 se propone una disminución en las condiciones de oxigenación de los sedimentos de fondo en el golfo de Panamá a partir de ca. 3.000 años AP.

La relación entre las formas epifaunales e infaunales que sirve como indicador de paleoxigenación muestra esta tendencia independiente del dominio de las formas infaunales a lo largo del núcleo (FIGURA 5). El análisis de susceptibilidad magnética del núcleo MC4 buscaba explorar si la disminución en las condiciones de paleoxigenación que se interpretan con los foraminíferos bentónicos responde a una mayor productividad superficial o a un mayor aporte de material terrígeno. Dado que los registros de susceptibilidad magnética en sedimentos de aguas profundas han sido usados como indicadores de paleo-precipitación (e.g. Robinson, 1990; Verosub and Roberts, 1995; Hounslow and Maher, 1999), se propone que la reducida variabilidad magnética detectada en el núcleo MC4 indicaría estabilidad en el aporte de material ferromagnético vía descarga fluvial y por consiguiente un régimen de precipitación constante (FIGURA 3).

Estos resultados contradicen interpretaciones previas para el Océano Pacifico oriental ecuatorial que sugieren condiciones tipo La Niña para el Holoceno medio (5.0008.000 años $\mathrm{AP}$ ) y una mayor recurrencia de eventos tipo El Niño desde los últimos 5.000 años hacia el presente, en particular para las Islas Galápagos (Bush and Colinvaux, 1994; Linsley et al., 1994; Riedinger et al., 2002; Lachniet et al., 2004; Conroy et al., 2008, Carré et al., 2012). La región del Darién, área fuente de los sedimentos del núcleo $\mathrm{MC} 4$, aunque reporta precipitaciones bajas en la temporada seca $(<500 \mathrm{~mm})$ no alcanzaría las condiciones de aridez necesarias para registrar un cambio en la susceptibilidad magnética, como si se ha observado en sitios próximos al Sahara o a la Península Arábica (e.g. Hounslow and Maher, 1999; Larrasoaña et al., 2008). Adicionalmente los cuerpos rocosos, de edad Cretácico Tardío a Oligoceno en esta región, son eminentemente vulcano-sedimentarios (e.g. Coates et al., 2004) y por tanto muy ricos en minerales ferromagnéticos, por lo que variaciones en la precipitación no afectarían su aporte al talud continental. Por tal motivo, los resultados de susceptibilidad magnética obtenidos del núcleo MC4 deben ser observados con cautela al momento de hacer inferencias en la paleoprecipitación del norte de la cuenca de Panamá. De esta forma para un futuro, se sugieren análisis complementarios para el MC4 como: (1) alquenonas ( $\mathrm{U}^{\mathrm{k}}{ }_{37}$ ), (2) el registro de $\mathrm{Mg}$ / $\mathrm{Ca}$ en foraminíferos planctónicos, (3) el radio entre los foraminíferos planctónicos Globorotalia menardii cultrata y Neogloboquadrina dutertertrei, (4) el índice de surgencia 
con radiolarios ("Upwelling radiolaria index") y, (5) la relación Florisphaera profunda/otros cocolitofóridos. Dichas estimaciones ya han sido probadas en la cuenca de Panamá y no solo permitirían la corroboración de las fluctuaciones en la paleoproductividad que detectaron los foraminíferos bentónicos, sino que también aclararían el rol que tendría el aporte de MO desde el continente (Martínez et al., 2006; Marín y Bayona, 2007; Pahnke et al., 2007; Rincón-Martínez et al., 2010; Patarroyo and Martínez, 2013a, 2013b, 2015).

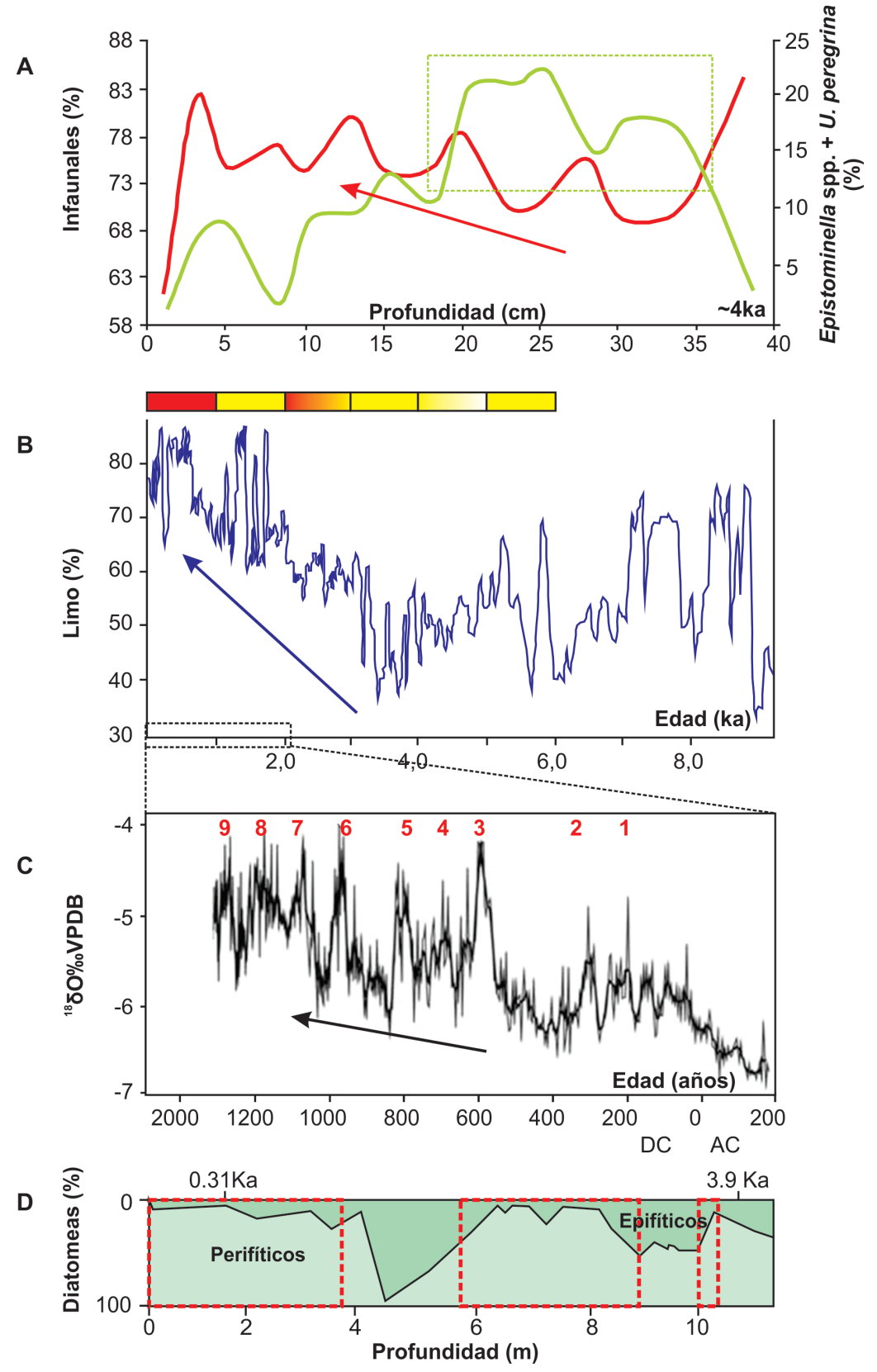

FIGURA 5. Comparación de la distribución de los foraminíferos bentónicos del núcleo KNR176-2-MC4 con algunos registros paleoclimáticos del Océano Pacifico ecuatorial oriental del Holoceno tardío. A. proporción de formas infaunales (línea roja) que aumenta hacia el presente, mientras que el recuadro verde indica una proporción $>10 \%$ en dos géneros indicadores de alta productividad. B. registro paleoclimático de las Islas Galápagos, donde el incremento del contenido de sedimentos tamaño limo hacia el presente es atribuido al aumento de la precipitación (Riedinger et al., 2002); los recuadros en color indican eventos de El Niño fuertes (rojo) y moderados (amarillo; Conroy et al., 2008). C. registro isotópico de la estalagmita de Chilibrillo (Panamá) donde se interpretan eventos fuertes de El Niño para los últimos 2000 años (números en rojo; Lachniet et al., 2004). D. registro de diatomeas del Lago Woodhouse (Darién) donde contenidos altos de diatomeas perifíticas son interpretados como periodos de precipitación baja (Bush and Colinvaux, 1994). 
A diferencia de los resultados de los análisis de susceptibilidad magnética, el cambio en la composición faunística en el núcleo MC4 hacia los 3.000 años AP ( $\sim 30 \mathrm{~cm}$ de profundidad) podría relacionarse con un aumento en la recurrencia del fenómeno ENSO. Para el Holoceno medio diversos registros paleoclimáticos apoyan la presencia de condiciones tipo La Niña en el norte de Suramérica (Carré et al., 2012). En cambio, en sedimentos lacustres de las Islas Galápagos (Lago Bainbridge) para el periodo 3.000-2.000 años AP hay evidencias de al menos 30 eventos de El Niño, los cuales se ven evidenciados por un aumento en la precipitación local. Dichos eventos superan en dos a tres órdenes de magnitud la recurrencia de eventos lluviosos fuertes ocurridos 6.000 años AP (Riedinger et al., 2002). De la misma forma, estudios sedimentológicos y geoquímicos efectuados en el lago El Junco, soportan una mayor recurrencia de eventos tipo El Niño desde los 4.000 años AP (FIGURA 5; Conroy et al., 2008).

Para los últimos 1.500 años la evidencia micropaleontológica no permite discernir más allá de condiciones de alta productividad como ocurre en el presente. Independientemente, la compilación de los estudios realizados en el norte de la cuenca de Panamá, sugieren una continuación en la recurrencia de periodos más secos hacia el presente, producto de eventos tipo El Niño (FIGURA 5; Bush and Colinvaux, 1994; Linsley et al., 1994; Piperno and Jones, 2003; Lachniet et al., 2004). Por tal razón, una mayor recurrencia de eventos tipo El Niño podría relacionarse con un aumento en la productividad superficial en el pasado reciente vía fenómenos de surgencia en el golfo de Panamá. Este patrón, que no ocurre con la zona de surgencia en el norte de Perú, obedece a las condiciones fisiográficas y oceanográficas que interactúan en el golfo a lo largo del año (Bishop et al., 1986; D'Croz and O'Dea, 2007; Reijmer et al., 2012). Sin embargo, al carecer el núcleo MC4 de un modelo de edad más robusto, la correlación de los eventos inferidos en este trabajo con los reportados por autores previos en la cuenca de Panamá, no implica que dichos eventos sean necesariamente coetáneos.

Por otro lado, evidencias de procesos tafonómicos para el núcleo MC-4 podrían ser interpretadas a través de la distribución de los foraminíferos aglutinados. Estos son particularmente abundantes en los centímetros superiores del núcleo, pero debido a su potencial de preservación bajo decrecen gradualmente hasta alcanzar nuevas proporciones en la base (FIGURA 4). Por ejemplo, formas tubulares de grano fino como
Rhabdammina son muy abundantes entre los 0 y $7 \mathrm{~cm}$. A su vez, el comportamiento errático en las abundancias de las formas porcelanáceas podría deberse a la disolución del carbonato, del cual algunas evidencias se observaron en el género Pyrgo. A pesar de que el núcleo MC4 fue colectado a una profundidad por encima de la lisoclina, el alto aporte de MO podría contribuir a incrementar los procesos de disolución (e.g. Martin, 1999). Por ende, los procesos tafonómicos dificultan la lectura del registro micropaleontológico en la parte superior del núcleo ya que índices como la diversidad y la equidad estarían influenciados por las fluctuaciones en las proporciones de los foraminíferos aglutinados y porcelanáceos (FIGURA 3).

A su vez, preferencias ambientales de las asociaciones de foraminíferos recientes en el núcleo MC4 afectarían la interpretación de la tanatocenosis. Esto es soportado por la abundancia alta a moderada, para el intervalo $0-10 \mathrm{~cm}$ de taxa como Globobulimina spp., Epistominella spp, C. bradyi, C. carinata y $O$. umbonatus. Estas especies son típicas de entornos eutróficos-mesotróficos y han sido reportadas en distintas zonas de surgencia (e.g. Gupta, 1997; Jorissen, 1999; Bernhard and Sen Gupta, 1999; Brüchert et al., 2000; Martins et al., 2006).

Las diferencias en sus proporciones reflejarían distintos hábitos de vida (e.g. epifaunal somero $=$ Epistominella; infaunal profundo $=$ Globobulimina $)$ los cuales presentan una relación estrecha con el contenido de oxígeno disuelto en los sedimentos de fondo y la disponibilidad de MO degradable (e.g. Bernhard and Sen Gupta, 1999; Gooday, 2003; Murray, 2006; Jorissen et al., 2007). Dado que este trabajo no distingue entre asociaciones vivas y muertas de foraminíferos, las variaciones en las abundancias de las taxa entre 0-10 cm serían un reflejo de: (1) cambios paleoambientales (e.g. mayor productividad superficial) y (2) nichos ecológicos recientes que favorecerían la predominancia de taxa infaunales profundas y someras (Kaminski et al., 1988; Gooday et al., 1992; Bernhard and Sen Gupta, 1999). Por tanto no se hacen inferencias paleoambientales para los 15 $\mathrm{cm}$ más superiores en el núcleo MC4.

\section{CONCLUSIONES}

Las asociaciones de foraminíferos bentónicos presentes en el núcleo KNR176-2-MC4 registran variaciones en la paleoproductividad y paleoxigenación del golfo de Panamá para los últimos 4.000 años. Para el intervalo entre ca. 4.000 y ca. 3.000 años AP se interpretan 
condiciones de oxigenación moderada en los sedimentos de fondo, en comparación con el reciente, cuando la productividad superficial se hace mayor y la oxigenación en los sedimentos de fondo se reduce. Organismos indicativos de alta productividad como Epistominella spp., U. peregrina y Trifarina son comunes a partir de los 3.000 años AP, remplazando formas como $U$. proboscidea y $O$. umbonatus. Sin embargo para la parte superior del núcleo, efectos tafonómicos y los distintos hábitats de vida de los foraminíferos enmascaran el registro, al igual que condicionan los índices de diversidad y equidad de las asociaciones.

A su vez, la recurrencia de períodos secos asociados al fenómeno ENSO para los últimos 5.000 años en el golfo de Panamá, tal como es propuesto en diversos estudios paleoclimáticos, vendría acompañada de una mayor intensidad y/o duración de los fenómenos de surgencia en esta región. Sin embargo, una disminución en los regímenes de precipitación no fue detectada en el registro de susceptibilidad magnética del núcleo MC-4, que en cambio si ha sido reportada en distintos registros paleoclimáticos del norte del Pacifico ecuatorial oriental. Análisis adicionales con otros indicadores (e.g. alquenonas, foraminíferos planctónicos) son propuestos para entender este contraste.

\section{AGRADECIMIENTOS}

Esta contribución fue realizada como parte de la tesis de Maestría en Ciencias de la Tierra (Universidad Eafit) del primer autor, y se encuentra enmarcada dentro del Programa de Jóvenes Investigadores de Colciencias y la Fundación para la Promoción de la Investigación y la Tecnología del Banco de la República (Proyecto 2915 "Transporte lateral de sedimentos de fondo de la cuenca de Panamá para el Cuaternario tardío: Estudio de las asociaciones de foraminíferos bentónicos y 230Th"). Agradecemos al profesor Lloyd Keigwin (Woods Hole Oceanographic Institution) por facilitar material del crucero KNR 176-2. Jackeline Dueñas (Universidad EAFIT) facilitó los resultados de susceptibilidad magnética del núcleo. Finalmente, los autores agradecen los comentarios y sugerencias hechas por los revisores.

\section{REFERENCIAS}

Akimoto, K. 1990. Distribution of recent benthic foraminiferal faunas in the Pacific of Southwest Japan and around Hachijojima Island. Science Reports of the Tohoku University Sendai (Geology), second series 60 (2): 288-291.
Akimoto, K. 1994. Cenozoic benthic foraminiferal biostratigraphy, paleobathymetry, paleoenvironments and paleoceanography of the New Hebrides Island Arc and north D'entrecasteaux ridge area. In: Greene, H.G., et al. (Eds.), Proc. ODP, Sci. Results, 134: College Proceedings of the Ocean Drilling Program. Scientific Results 134, pp: 265-291.

Bakun, A. 1990. Global climate change and intensification of coastal ocean upwelling. Science, 247: 198-201.

Bandy, O.L., and Arnal, R.E. 1957. Distribution of Recent foraminifera off west coast of Central America. AAPG Bulletin, 41: 2037-2053.

Bandy, O.L., and Rodolfo, K.S. 1964. Distribution of foraminifera and sediments, Peru-Chile trench area. Deep Sea Research, 11: 817-837.

Bernhard, J.M., and Sen Gupta, B.K. 1999. Foraminifera of oxygen-depleted environments. In: Sen Gupta, B.K. (Ed.), Modern Foraminifera. Kluwer Academic Publishers, Londres, pp. 201-216.

Bernhard, J.M., and Reimers, C.E. 1991. Benthic foraminiferal population fluctuations related to anoxia: Santa Barbara Basin. Biogeochemistry, 15: 127-149.

Betancur, M.A., y Martínez, J.I. 2003. Foraminíferos bentónicos recientes en sedimentos de fondo de la cuenca de Panamá (Pacífico Colombiano), como indicadores de productividad y oxigenación. Boletín de Investigaciones Marinas y Costeras, 32: 3-18.

Bishop, K.B., Stepien, J.C., and Wiebe, P.H. 1986. Particulate matter distribution and flux in the Panama basin: response to environmental forcing. Progress in Oceanography, 17 (1-2): 1-59.

Bolli, H.M., Beckmann, J.P., and Saunders, J.B. 1994. Benthic Foraminiferal biostratigraphy of the South Caribbean region. Cambridge University Press, $411 \mathrm{p}$.

Boltovskoy, E., y Gualancañay, E. 1975. Foraminíferos Bentónicos Actuales de Ecuador. 1.- Provincia Esmeraldas. Instituto Oceanográfico Biológico, 5: 1-56.

Bornmalm, L. 1997. Taxonomy and paleoecology of Late Neogene benthic foraminifera from the Caribbean Sea and Eastern Equatorial Pacific Ocean. Fossils and Strata 41: 1-96. 
Bornmalm, L., Widmark, J.G.V., and Malmgren, B.A. 1999. Changes in circulation and trophic levels in the Pliocene Caribbean Sea: Evidence from benthic foraminifer accumulation rates. Journal of Foraminiferal Research, 29 (3): 209-221.

Bradley, R.S. 1999. Paleoclimatology: Reconstructing Climate of the Quaternary. Academic Press, Londres, 613p.

Brüchert, V., Pérez, M.E., and Lange, C.B. 2000. Coupled primary production, benthic foraminiferal assemblages, and sulfur diagenesis in organic-rich sediments of the Benguela upwelling system. Marine Geology, 163: 27-40.

Bush, M.B., and Colinvaux, P.A. 1994. Tropical forest disturbance: Paleoecological records from Darien, Panama. Ecology, 75: 1761-1768.

Buzas, M.A., and Gibson, T.G. 1969. Species diversity: benthonic foraminifera in western North Atlantic. Science, 163, 72-75.

Carré, M., Azzoug, M., Bentaleb, I., Chase, B.M., Fontugne, M., Jackson, D., Ledru, M.P., Maldonado, A., Sachs, J.P., and Schauer, A.J. 2012. Mid-Holocene mean climate in the southeastern Pacific and its influence on South America. Quaternary International, 253: 55-66.

Chelton, D.B., Freilich, M.H., and Esbensen, S.K. 2000. Satellite observations of the wind jets off the Pacific Coast of Central America. Part II: Relationships and dynamical considerations, Monthly Weather Review, 128: 2019-2043.

Clark, J.P. 1988. Panama, Pacific Coast: Gulf of Panama, Nautical Map. Admiralty Charts and Publications, Taunton, Reino Unido.

Clark, F.E., and Patterson, R.T. 1993. An illustrated key to the identification of unilocular genera of calcareous foraminifera. Journal of Paleontology, 67 (1): 20-28.

Coates, A.G., Collins, L.S., Aubry, M.P., and Berggren, W.A. 2004. The Geology of the Darien, Panama, and the late Miocene-Pliocene collision of the Panama arc with northwestern South America. Geological Society of America Bulletin, 116: 1327-1344.

Conroy, J.L., Overpeck, J.T., Cole, J.E., Sanan, T.M., and Steinitz-Kannan, M. 2008. Holocene changes in eastern tropical Pacific climate inferred from a Galápagos lake sediment record. Quaternary Science Reviews, 27: 1166-1180.
Cushman, J.A. 1920. The foraminifera of the Atlantic Ocean, Part 2 Lituolidae. Smithsonian Institution Bulletin 84: 1-111.

Cushman, J.A. 1927. Recent foraminifera from off the west coast of America. Scripps Institution of Oceanography Technical Series, 1: 119-188.

Cushman, J.A. 1933. Some new foraminiferal genera. Contributions from the Cushman Laboratory for Foraminiferal Research, 9: 32-38.

Cushman, J.A., and McCulloch, I. 1942. Some Virgulininae in the collections of the Allan Hancock Foundation. Allan Hancock Pacific Expedition, 6 (2): 179-230.

D’Croz, L., Del Rosario, J.B., and Gómez, J.A. 1991. Upwelling and phytoplankton in the Bay of Panama. Revista de Biología Tropical, 39: 233-241.

D'Croz, L., and O'Dea, A.O. 2007. Variability in upwelling along the Pacific shelf of Panama and implications for the distribution of nutrients and chlorophyll. Estuarine and Coastal Shelf Science, 73: 325-340.

De, S., and Gupta, A.K. 2010. Deep-sea faunal provinces and their inferred environments in the Indian Ocean based on distribution of recent benthic foraminifera. Palaeogeography Palaeoclimatology Palaeoecology, 291: 429-442.

Douglas, R.G. 1973. Benthonic foraminiferal biostratigraphy in the Central North Pacific, Leg 17. In: Winterer, E.L. (Ed.), Deep Sea Drilling Project, Initial Reports, 17, pp. 607-672.

Dunbar, R.B. 1983. Stable isotope record of upwelling and climate from Santa Barbara Basin, California Coastal Upwelling. In: Thiedel, J., Suess, E. (Eds.), Its Sediment Record, pp. 217-246.

Earland, A. 1934. Foraminifera: Part III - the Falklands sector of the Antarctic (excluding South Georgia). Discovery Reports, 10: 1-208.

Fiedler, P.C., and Talley, L.D. 2006. Hydrography of the eastern tropical Pacific: A review. Progress in Oceanography, 69: 143-180.

Fiorini, F. 2015. Recent benthic foraminifera from the Caribbean continental slope and shelf off west of Colombia. Journal of South American Earth Sciences, 60: 117-128. 
Fontanier, C., Jorissen, F.J., Licari, L., Alexandre, A.P., Anschutz, P., and Carbonel, P. 2002. Live benthic foraminiferal faunas from the Bay of Biscay: faunal density, composition, and microhabitats: Deep Sea Research, I 49: 751-785.

Forsbergh, E.D. 1969. On the climatology, oceanography and fisheries of the Panama Bight. Bulletin of the InterAmerican Tropical Tuna Commission, 14: 49-259.

Freudenthal, T., Meggers, H., Henderiks, J., Kuhlmann, H., Moreno, A., and Wefer, G. 2002. Upwelling intensity and filament activity off Morocco during the last 250,000 years. Deep-Sea Research, II 49: 3655-3674.

Golik, A. 1968. History of the Holocene transgression in the Gulf of Panama. Journal of Geology, 76 (5): 497-507.

Golik, A., and Phleger, F.B. 1977. Benthonic foraminifera from the Gulf of Panama. Journal of Foraminiferal Research, 7: 83-99.

Gooday, A.J. 2003. Benthic foraminifera (Protista) as tools in deep-water palaeoceanography: Environmental influences on faunal characteristics. Advances in Marine Biology, 46: 1-90.

Gooday, A.J., Levin, L.A., Linke, P., and Heeger, T. 1992. The role of benthic foraminifera in deep-sea food webs and carbon cycling. In: Rowe G.T., Pariente, V. (Eds.), Deep-Sea Food Chains and the Global Carbon Cycle, pp. 63-91.

Gualancañay, E. 1983. Foraminíferos bentónicos del Golfo de Guayaquil. Acta Oceanográfica del Pacífico, 2 (2): 589-657.

Gualancañay, E. 1986. Foraminíferos del Rift de Galápagos. Acta Oceanográfica del Pacífico, 3 (1): 149-155.

Gupta, A.K. 1994. Taxonomy and bathymetric distribution of Holocene deep-sea benthic foraminifera in the Indian Ocean and the Red Sea. Micropaleontology, 40: 351-367.

Gupta, A.K. 1997. Paleogeographic and paleoclimatic history of the Somali Basin during the PliocenePleistocene; Multivariate analyzes of benthic foraminifera Site 241. Journal of Foraminiferal Research, 27 (3): 196-208.
Hammer, O., Harper, D.A.T., and Ryan, P.D. 2001. PAST: paleontological statistics software package for education and data analysis. Palaeontología Electrónica, 4 (1): http://palaeo-electronica.org/2001_1/past/ issue1_01.htm.

Hasegawa, S., Sprovieri, R., and Poluzzi, A. 1990. Quantitative analysis of benthic foraminiferal assemblages from Plio-Pleistocene sequences in the Tyrrhenian Sea, ODP Leg 107. In: Kastens, K.A., et al. (Eds.) Proceedings of the Ocean Drilling Program. Scientific Results, 107, pp. 461-478.

Hayek, L.A.C., and Buzas, M.A. 1997. Surveying Natural Populations. Columbia University Press, Nueva York, 562p.

Holbourn, A.E., and Henderson, A.S. 2002. Reillustration and Revised Taxonomy for Selected Deepsea Benthic Foraminifers. Palaeontología Electrónica 4 (2): http://palaeo-electronica.org/paleo/2001_2/foram/ issue2_01.htm.

Hounslow, M.W., and Maher, B.A., 1999. Source of the climate signal recorded by magnetic susceptibility variations in Indian Ocean sediments. Journal of Geophysical Research, 104: 5047-5061.

Jones, R.W. 1994. The Challenger Foraminifera. Oxford University Press, Oxford, 149p.

Jorissen, F.J. 1999. Benthic foraminiferal microhabitats below the sediment-water interface. In: Sen Gupta, B.K. (Ed.), Modern Foraminifera. Kluwer Academic Publishers, Londres, pp. 161-179.

Jorissen, F.J., Fontanier, C., and Thomas, E. 2007. Paleoceanographical indicators based on deep-sea benthic foraminiferal assemblage characteristics. Developments in Marine Geology, 1: 263-325.

Kaiho, K. 1992. Eocene to Quaternary benthic foraminifers and paleobathymetry of the Izu-Bonin Arc, Legs 125 and 126. In: Taylor, B., et al. (Eds.), Proceedings of the Ocean Drilling Program, Scientific Results, 126, pp. 285-310.

Kaiho, K., and Nishimura, A. 1992. Distribution of Holocene benthic foraminifers in the Izu-Bonin Arc. In: Taylor, B., et al. (Eds.), Proceedings of the Ocean Drilling Program, Scientific Results, 126, pp. 311-320. 
Kaminski, M., Grassle, J.F., and Whitlatch, R.B. 1988. Life history and recolonization among agglutinated foraminifera in the Panama Basin. Abhandlungen der Geologischen. Bundensanstalt, 41: 229-243.

Kender, S., Kaminski, M.A., and Jones, R.W. 2008. Oligocene Deep-Water Agglutinated Foraminifera from the Congo Fan, Offshore Angola: Palaeoenvironments and Assemblage Distributions. In: Kaminski, M.A. and Coccioni, R. (Eds.), Proceedings of the Seventh International Workshop on Agglutinated Foraminifera. Grzybowski Foundation Special Publication 13, pp. 107-156.

Lachniet, M.S., Burns, S.J., Piperno, D.R., Asmerom, Y., Polyak, V.J., Moy, C.M., and Christenson, K. 2004. A 1500-year El Niño/Southern Oscillation and rainfall history for the Isthmus of Panama from speleothem calcite. Journal of Geophysical Research, 109: D20117, doi:10.1029/2004JD004694.

Lalicker, C.G., and McCulloch, I. 1940. Some Textulariidae of the Pacific Ocean. Allan Hancock Pacific Expeditions, 6 (2): 115-143.

Larrasoaña, J.C., Roberts, A.P., and Rohling, E.J. 2008. Magnetic susceptibility of eastern Mediterranean marine sediments as a proxy for Saharan dust supply?. Marine Geology, 254: 224-229.

Lemos, R.T., and Pires, H.O. 2004. The upwelling regime off the West Portuguese coast, 1941-2000, International Journal of Climatology, 24: 511-524.

Licari, L.N., and Mackensen, A. 2005. Benthic Foraminifera off West Africa ( $18 \mathrm{~N}$ to $32 \mathrm{~S}$ ): Do Live Assemblages from the Topmost Sediment reliably record Environmental Variability?. Marine Micropaleontology, 55: 205-233.

Linsley, B.K., Dunbar, R.B., Wellington, G.M., and Mucciarone, D.A. 1994. A coral-based reconstruction of Intertropical Convergence Zone variability over Central America since 1707, Journal of Geophysical Research, 99: 9977-9994.

Loeblich, A.R., and Tappan, H. 1964. Sarcodina and Foraminifera. In: Moore, R.C. (Ed.), Treatise on Invertebrate Paleontology, Part C, Protista, 2. Kansas University and Geological Society of America, Lawrence, 936p.

Lonsdale, P., and Malfait, B.T. 1974. Abyssal dunes of foraminiferal sand on the Carnegie Ridge. Geological Society of America Bulletin, 85: 1697-1712.
Loubere, P. 1991. Deep-sea benthic foraminiferal assemblage response to a surface ocean productivity gradient: A test. Paleoceanography, 6 (2): doi: 10.1029/90PA02612.

Loubere, P. 1999. A multiproxy reconstruction of biological productivity and oceanography in the eastern equatorial Pacific for the past 30,000 years, Marine Micropaleontology, 37: 173-198.

Lyle, M. 1992. Composition maps of surface sediments of the eastern tropical Pacific Ocean. In: Mayer, L., et al., (Eds.), Proceedings of the Ocean Drilling Program, Initial Reports, 138, pp. 101-115.

Mackensen, A., Grobe, H., Kuhn, G., and Fütterer, D.K. 1990. Benthic foraminiferal assemblages from the eastern Weddell Sea between 68 and $73^{\circ} \mathrm{S}$ : distribution, ecology and fossilization potential. Marine Micropaleontology, 16: 241-283.

Magurran, A.E. 2004. Measuring Biological Diversity. Blackwell Publishers, Oxford, 260p.

Marín, D., y Bayona, G. 2007. Foraminíferos y radiolarios holocénicos de la Cuenca de Panamá. Proyecto de Grado. Departamento de Geología, Universidad EAFIT, Medellín.

Martin, R.E. 1999. Taphonomy and temporal resolution of foraminiferal assemblages. In: Sen Gupta, B. (Ed.), Modern Foraminifera. Kluwer Academic Publishers, Londres, pp. 281-298.

Martínez, J.I., Rincon, D., Yokoyama, Y., and Barrows, T.T. 2006. Foraminifera and Coccolithophorid Assemblage Changes in the Panama Basin during the Last Deglaciation: Response to Sea-surface Productivity Induced by a Transient Climate Change. Paleogeography Palaeoclimatology Paleoecology, 234: 114-126.

Martins, V., Jouanneau, J.M., Weber, O., and Rocha, F. 2006. Tracing the late Holocene evolution of the NW Iberian upwelling system. Marine Micropaleontology, 59: 35-55.

Matoba, Y., and Yamaguchi, A. 1982. Late Pliocene to Holocene benthic foraminifers of the Guaymas Basin, Gulf of California: Sites 477 through 481. In: Curray, J.R., et al. (Eds.), Initial Reports of the Deep Sea Drilling Project 64 (2), pp. 1027-1052.

McDougall, K. 1985. Miocene to Pleistocene benthic foraminifers and paleoceanography of the Middle America Slope, Deep Sea Drilling Project Leg 84. In: von Huene, R. et al. (Eds.). Initial Reports of the Deep Sea Drilling Project, 84: 363-418. 
Moore, J.R., Heath, G.R., and Kowsman, R.O. 1973. Biogenic sediments of the Panama Basin. Journal of Geology, 81: 458-473.

Murray, J.W. 2006. Ecology and Applications of Benthic Foraminifera. Cambridge University Press, Cambridge, 426p.

Narayan, N., Paul, A., Mulitza, S., and Schulz, M. 2010. Trends in coastal upwelling intensity during the late 20th century. Ocean Science Discussions, 7: 335-360.

Nomura, R. 1992. Miocene benthic foraminifers at Sites 794, 795 and 797 in the Sea of Japan with reference to the foram sharpline in the Honshu Arc. In: Suess, E., et al. Proceedings of the Ocean Drilling Program 127/128 part 1, pp. 493-540.

Nisha, N.R. 2002. Foraminiferal and pteropod records in the late Quaternary sequence off Northern Kerala and their oceanographic and climatic significance. $\mathrm{PhD}$ Thesis, Cochin University, India.

Ohkushi, K., Thomas, E., and Kawagata, H. 2000. Abyssal benthic foraminifera from Northwestern Pacific (Shatsky Rise) during the last 298 kyr. Marine Micropaleontology, 38: 119-147.

Pahnke, K., Sachs, J.P., Keigwin, L., Timmermann, A., and Xie, S.P. 2007. Eastern tropical Pacific hydrologic changes during the past 27,000 years from $\mathrm{D} / \mathrm{H}$ ratios in alkenones. Paleoceanography, 22: PA4214, doi:10.1029/2007PA001468.

Parr, W.J. 1950. Foraminifera: British, Australian and New Zealand Antarctic Research Exped. 1929-1931, Repts. ser. B (Zoology and Botany), 5 (6): 233-392.

Patarroyo, G., y Martínez, J.I. 2013a. Foraminíferos bentónicos recientes en las aguas profundas de la cuenca de Panamá: Ecología y su posible relación con las corrientes de fondo. Boletín de Investigaciones Marinas y Costeras, 42 (1): 33-58.

Patarroyo, G., and Martínez, J.I. 2013b. Deep sea benthic foraminifera distribution and environmental preferences in Panama Basin. Caldasia, 35 (2): 311-324.

Patarroyo, G., and Martínez, J.I. 2015. Late quaternary sea bottom conditions in the southern Panama basin, Eastern Equatorial Pacific. Journal of South American Earth Sciences, 6: 346-359.

Pauly, D., and Christensen, V. 1995. Primary production required to sustain global fisheries. Nature, 374: 255-257.
Piperno, D.R., and Jones, J. 2003. Paleoecological and archaeological implications of a Late Pleistocene/Early Holocene record of vegetation and climate from the Pacific coastal plain of Panama. Quaternary Research, 59: 79-87.

Poveda, G., Waylen, P.R., and Pulwarty, R.S. 2006. Annual and interannual variability of the present climate in northern South America and southern Mesoamerica. Palaeogeography Palaeoclimatology Palaeoecology, 234: 3-27.

Rathburn, R., and Corliss, B.H. 1994. The ecology of living (stained) deep-sea benthic foraminifera from the Sulu Sea. Paleoceanography, 9: 87-150.

Reijmer, J.J.G., Bauch, T., and Schäfer, P. 2012. Carbonate facies patterns in surface sediments of upwelling and non-upwelling shelf environments (Panama, East Pacific). Sedimentology, 59: 32-56.

Revets, S.A. 1996. The generic revision of five families of Rotaliine foraminifera. Cushman Foundation for Foraminiferal Research. Special Publication, 34: 1-113.

Revets, S.A. 2005. A key to the unilocular hyaline Foraminifera. Journal of Micropalaeontology, 24: 145-158.

Riedinger, M.A., Stinitz-Kannan, M., Last, W.M. and Brenner, M. 2002. A $610014 \mathrm{C}$ record of El Niño activity from the Galápagos Islands. Journal of Paleolimnology, 27: 1-7.

Rincón-Martínez, D., Lamy, F., Contreras, S., Leduc, G., Bard, E., Saukel, C., Blanz, T., Mackensen, A., and Tiedemann, R. 2010. More humid interglacials in Ecuador during the past $500 \mathrm{kyr}$ linked to latitudinal shifts of the equatorial front and the Intertropical Convergence Zone in the eastern tropical Pacific. Paleoceanography, 25: PA2210, doi:10.1029/2009PA001868.

Robinson, S.G. 1990. Applications for whole-core susceptibility measurements of deep sea sediments: ODP Leg 115 results. En: Duncan, R.A., et al. (Eds.), Proceedings of the Ocean Drilling Program, Scientific Results, 115: 737-771.

Rodríguez-Rubio, Schneider, E.W., and Abarca del Rio, R. 2003. On the seasonal circulation within the Panama Bight derived from satellite observations of wind, altimetry and sea surface temperature. Geophysical Research Letters, 30: 1410, doi:10.1029/2002GLO16794. 
Sarkar, S., De, S., and Gupta, A.K. 2009. Late Quaternary benthic foraminifera from Ocean Drilling Program Hole 716A, Maldives Ridge, southeastern Arabian Sea. Micropaleontology, 55 (1): 23-48.

Shannon, C.E. 1948. A mathematical theory of communication: Bell System Technical Journal 27, 379-423.

Schönfeld, J., and Spiegler, D. 1995. Benthic foraminiferal biostratigraphy site 861, Chile triple junction, Southeastern Pacific. In: Lewis, S.D., et al. (Eds.), Proceedings of the Ocean Drilling Program, 141, pp. 213-221

Sen Gupta, B. 1999. Modern Foraminifera. Kluwer Academic Publishers, Londres, 371p.

Smayda, T.J. 1963. A quantitative analysis of the phytoplankton of the Gulf of Panama I. Results of the regional phytoplankton surveys during July and November, 1957 and March, 1958. Bulletin of the Inter-American Tropical Tuna Commission, 7: 191-253.

Smith, P.B. 1963. Quantitative and qualitative analysis of the Family Bolivinidae. United States Geological Survey, Professional Paper, 429-A: A1-A39.

Smith, P.B. 1964. Ecology of benthonic species: Recent foraminifera off Central America. United States Geological Survey, Professional Paper, 429- B: B1-B55.

Swift, S.A. 1977. Holocene rates of sediment accumulation in the Panama Basin, Eastern Equatorial Pacific: pelagic sedimentation and lateral transport. The Journal of Geology, 85 (3): 301-319.

Thomas, E. 1985. Late Eocene to recent deep-sea benthic foraminifera from the central equatorial Pacific Ocean. In: Mayer, L., et al. (Eds.), Deep Sea Drilling Project, Initial Reports, 85, pp. 650-694.

Vázquez-Riveiros, N., and Patterson, T. 2008. An illustrated guide to fjord foraminifera from the SeymourBelize inlet complex, Northern British Columbia, Canada. Paleontología Electrónica, 11 (1): http://palaeoelectronica.org/2008_1/145/index.html.

Verosub, K.L., and Roberts, A.P. 1995. Environmental magnetism: past, present and future. Journal of Geophysical Research, 100: 2175-2192.
Walsh, J.J. 1991. Importance of continental margins in the marine biogeochemical cycling. Nature, 350: 53-55.

Wright, R., College, B., and Wisconsin, B. 1978. Neogene benthic foraminifers from DSDP Leg 42A, Mediterranean Sea. In: Hsü, K.J., et al. (Eds.), Deep Sea Drilling Project Initial Reports 42 Part 1, pp. 709-726.

Xia, T., and Fan, Z. 1996. A study of foraminifera in the core NP93-2 from the Prydz Bay, Antarctica. Antarctic Research, 7 (2): 126-140.

Xie, S.P., Xu, H., Kessler, W.S., and Masami, N. 2005. Air-sea interaction over the eastern Pacific warm pool: Gap winds, thermocline dome, and atmospheric convection. Journal of Climate, 18 (1): 5-20.

Trabajo recibido: septiembre 9 de 2015

Trabajo aceptado: enero 22 de 2016

Manuscrito publicado en internet: enero 25 de 2016 
ANEXO. Lista de las principales especies encontradas en el núcleo KNR176-2-MC4.

Ammobaculites agglutinans: Akimoto (1990), p. 226, pl. 12, fig. 4.

Ammobaculites americanus: Cushman (1920), p. 64, pl. 12, figs. 6-7.

Anomalinoides globulosus: Thomas (1985), p. 691, pl. 12, figs. 6-7.

Astrononion gallowayi: Vázquez-Riveiros and Patterson (2008), fig. 13 (1a-c).

Bolivina humilis: Matoba and Yamaguchi (1992), p. 1053, pl. 1, figs. 6A-B.

Bolivina minuta: Smith (1963), p. A19-20, pl. 30, figs. 11-12.

Bolivina pacifica: Matoba and Yamaguchi (1982), p. 1053, pl. 1, figs. 8-9.

Bolivina plicata: Gualancañay (1983), p. 631, pl. 6, fig. 8.

Bolivina seminuda: Bornmalm (1997), p. 45, fig. 18e.

Bolivina semiperforata: Smith (1963), p. A19, pl. 30, figs. 5-8.

Bolivina subadvena: Matoba and Yamaguchi (1982), p. 1053, pl. 1, figs. 15-17.

Brizalina argentea: Matoba and Yamaguchi (1982), p. 1053, pl. 1, figs. 18a-19b.

Brizalina interjuncta bicostata: Matoba y Yamaguchi (1982), p. 1053, pl. 1, fig. 21.

Bulimina barbata: Kaiho (1992), p. 306, pl. 4, fig. 8.

Bulimina costata: Sarkar et al. (2009), p. 29, pl. 2, fig. 9.

Bulimina denudata: Smith (1964), pl. 2, fig. 12.

Bulimina marginata: Bornmalm (1997), p. 45, fig. $18 \mathrm{i}$.

Bulimina pagoda: Matoba and Yamaguchi (1982), p. 1054, pl. 2, fig. 3.

Bulimina rostrata: Akimoto (1990), p.236, pl. 16, fig. 7

Bulimina spinifera: Globobulimina spinifera para Cushman (1927), p. 151, pl 2, fig. 15.

Bulimina striata: Akimoto (1990), pl. 16, fig. 8.

Buliminella curta: Matoba and Yamaguchi (1982), p. 1053, pl. 1, fig. 2.

Buliminella elegantissima: Sarkar et al. (2009), p. 29, pl. 2, fig. 13.

Cancris sagra: Gualancañay (1983), p. 623, pl. 2, figs. 8a-b.

Cassidulina carinata: Akimoto (1990), pl. 17, fig. 3; Kaiho (1992), p. 305, pl. 3, fig. 4.

Cassidulina crassa: Gualancañay (1983), p. 629, pl. 5, fig. 13.

Chilostomella oolina: Fiorini (2015), p. 126, pl. 3, fig. 13.

Chilostomella ovoidea: Kaiho and Nishimura (1992), p. 319, pl. 4, fig. 16.

Cibicidoides brady: Ohkushi et al. (2000), p. 142, pl. 4, figs. 6a-c.

Cibicidoides lobatulus: C. lobatulus para Houlbourn and Henderson (2002), p. 17, figs. 3 (1-3).

Cibicidoides mundulus: Bornmalm (1997), p. 75, figs. 26G-I.

Cibicidoides robertsonianus: Houlbourn and Henderson (2002), p. 20, figs. 4 (10-12).

Cibicidoides wuellerstorf: Houlbourn and Henderson (2002), p. 24, figs. 5 (6-8).

Cyclammina cancellata: Kender et al. (2008), p. 542, pl. 9, figs. 5-6.

Cyclammina pussilla: Cushman (1920), p. 56, pl. 11, figs. 4-6.

Eggerella bradyi: Houlbourn and Henderson (2002), pags. 8, 10, fig. 2 (4-5).

Ehrenbergina pacifica: Kaiho (1992), p. 305, pl. 3, figs. 19a-c.

Epistominella exigua: Ohkushi et al. (2000), p.141, pl. 3, figs. 1a-c.

Epistominella pacifica: Schönfeld and Spiegler (1995), p. 222, pl. 2, figs. 8-9.

Epistominella smithi: Matoba and Yamaguchi (1982), p. 1055, pl. 3, figs. 1A-C.

Evolcassidulina brevis: Akimoto (1990), p. 236, pl. 17, fig. 5.

Evolcassidulina tenuis: Ohkushi et al. (2000), p. 138, pl. 1, fig. 8.

Fissurina abyssicola: Ohkushi et al. (2000), p. 138, pl. 1, fig. 7.

Fissurina bradyi: Kaiho (1992), p. 304, pl. 2, fig. 11.

Fissurina fimbriata: Bornmalm (1997) p. 42, figs. 17a-b.

Fissurina kerguelensis: Bornmalm (1997), p. 42, fig. 17c.

Fissurina marginata: Bornmalm (1997), p. 42, fig. 17d-f.

Fissurina semimarginata: Bornmalm (1997), p. 42, fig. 17g.

Fissurina wiesneri: Bornmalm (1997), p. 42, fig. 17h-i.

Fursenkoina complanata: Wright et al. (1978), p. 722, pl. 4, fig. 17.

Fursenkoina cornuta: Matoba and Yamaguchi (1982), p. 1055, pl. 3, figs. 8a-b. 
Fursenkoina rotundata: Sarkar et al. (2009), p. 33, pl. 4, fig. 15. Globobulimina affinis: Bornmalm (1997), p. 50, figs. 19a-b. Globobulimina auriculata: Bornmalm (1997), p. 50, fig. 19c. Globobulimina ovula: Hasegawa et al. (1990), p. 476, pl. 3, fig. 18.

Globobulimina pacifica: Matoba and Yamaguchi (1982), p. 1054, pl. 2, fig. 8.

Globobulimina pupoides: Nomura (1992), p. 539, pl. 3, figs. 1-2.

Globocassidulina subglobosa: Hasegawa (1990), p. 477, pl. 4, figs. 5-6.

Gyroidina altiformis: Bornmalm (1997), p. 70, figs. 24D-F.

Gyroidina neosoldani: Gyroidina cf. neosoldani para Hasegawa et al. (1990), p. 478, pl. 5, figs. 10-12.

Gyroidina soldani: Akimoto (1994), p. 290, pl. 3, figs. 3a-c.

Haplophragmoides sphaeriloculum: Cushman (1920) p. 45, pl. 8, fig. 3.

Hoeglundina elegans: Bornmalm (1997), p. 45, figs. 18C-D.

Hormosinella distans: Kaiho and Nishimura (1992), p. 316, pl. 1, figs. 11-12.

Lagena elongata: Gualancañay (1983), p. 633, pl. 7, fig. 16.

Lagena gracilisima: Gualancañay (1983), p. 633, pl. 7, figs. 15a-b.

Lagena hispidula: Nisha (2002), p.143, pl. 2, fig. 11.

Lagena laevis: Kaiho and Nishimura (1992), p. 318, pl. 3, fig. 8.

Lagena striata: McDougall (1985), p. 414, pl. 2, fig. 4; Nisha (2002), p. 143, pl. 2, figs. 12-13.

Laticarinina pauperata: Bornmalm (1997), p. 58, figs. 21a-c; Fiorini (2015), p. 126, pl. 3, fig. 1.

Lagenammina atlantica: Ohkushi et al. (2000), p. 138, pl. 1, figs. 2a-b.

Lagenammina difflugiformis: Mackensen et al. (1990), pl. 6, fig. 9.

Lenticulina atlantica: Bornmalm (1997), p. 34, figs. 15g-h.

Loxostomum pseudobeyrichi: Matoba and Yamaguchi (1982), p. 1053, pl. 1, figs. 25A-B.

Marginulina obesa: M. glabra cf. obesa para Bolli et al. (1994), p. 295, fig. 77 (24).

Martinottiella communis: Kaiho and Nishimura (1992), p. 317, pl. 2, fig. 13.

Martinottiella nodulosa: Cushman (1933) p. 32-38.

Melonis affinis: Bornmalm (1997), p. 78, Fig. 27D-F.

Melonis barleanum: Gupta (1994), pl. 6, fig. 1.

Melonis pompilioides: Kaiho (1992), p. 309, pl. 7, figs. 11a-b.

Nonion gernanicum: Bornmalm (1997), p. 65, figs. 23e-g.

Nonionella atlantica: Gualancañay (1983), p. 623, pl. 2, fig. 1.

Nonionella basiloba: Matoba and Yamaguchi (1982), p. 1056, pl. 4, figs. 3A-B.

Nuttallides umbonifera: Thomas (1985), p. 692, pl. 13, figs. 1-2.

Oolina desmophora: Bornmalm (1997), p. 42, fig. 17k.

Oolina globosa: Ohkushi et al. (2000), p. 138, pl. 1, figs. 11a-b.

Oolina hexagona: Nomura (1992), p. 539, pl. 3, fig. 9.

Oridorsalis umbonatus: Matoba and Yamaguchi (1982), p. 1056, pl. 4, figs. 11a-c.

Osangularia interrupta: Douglas et al. (1973), p. 643, pl. 11, figs. 1-3.

Parafissurina lateralis: Ohkushi et al. (2000), p. 138, pl. 1, figs. 12a-c.

Parafissurina sublata: Bornmalm (1997), p. 45, fig. 18a.

Paratrochammina challengeri: Houlbourn and Henderson (2002), p. 13, fig. 2 (8-10).

Planulina exorna: McDougall (1985), p. 417, pl. 5, fig. 6.

Pullenia bulloides: Ohkushi et al. (2000), p. 143, pl. 5, figs. 9a-b.

Pullenia quinqueloba: Akimoto (1994), p. 288, pl. 1, figs. 4a-b.

Pyrgo depressa: Jones (1994), pl.2, figs.12, 16-17; Nisha (2002), p. 141, pl. 1, fig. 27.

Pyrgo lucernula: Kaiho (1992), p. 304, pl. 2, fig. 6; Sarkar et al. (2009), p. 39; pl. 7, fig. 20.

Pyrgo murhyna: Bornmalm (1997), p. 27, fig. 14B; Fiorini (2015), p. 125, pl. 2, figs. 4-5.

Pyrgo oblonga: Ohkushi et al. (2000), p. 138, pl. 1, figs. 4a-c.

Pyrgo serrata: Houlbourn and Henderson (2002), p. 24, figs. 5 (9-11).

Quinqueloculina lamarckiana: Kaiho (1992), p. 304, pl. 2, fig. 2.

Quinqueloculina venusta: Bornmalm (1997), p. 23, figs. 13k-1.

Quinqueloculina weaveri: Sarkar et al. (2009), p. 41, pl. 8, figs. 9-10.

Recurvoides contortus: Earland (1934), p. 91, pl. 10, figs. 7-19. 
Reophax dentaliniformis: Kaiho and Nishimura (1992), p. 316, pl. 1, fig. 16.

Reophax guttifer: Kaiho and Nishimura (1992), p. 316, pl. 1, fig. 13.

Reophax pilulifer: Kender et al. (2008), p. 140, pl. 5, figs. 3-4.

Reophax scorpiurus: Kaiho and Nishimura (1992), p. 316, pl. 1, figs. 14-15.

Rhabdammina abyssorum: Akimoto (1990), pags. 226, 230, pl. 12, fig. 10, pl. 14, fig. 4.

Saccammina sphaerica: Akimoto (1990), p. 226, pl. 12, fig. 1.

Spiroloculina tenuis: Kender et al. (2008), p. 549, pl. 14, fig. 2.

Sugrunda eckisi: Matoba and Yamaguchi (1982), p. 1055, pl. 3, figs. 10A-B.

Textularia agglutinans: Lalicker and McCulloch (1940), p. 123, pl. 13, figs. 2a-c.

Textularia articulata: Lalicker and McCulloch (1940), p. 118, pl. 13, figs. 3a-d.

Textularia fistula: Lalicker and McCulloch (1940), p. 127, pl. 14, fig. 10.

Textularia scrupta: Lalicker and McCulloch (1940), p. 141, pl. 16, fig. 25.

Trifarina angulosa: Sarkar et al. (2009), p. 45, pl. 10, fig. 11.

Trifarina bradyi: Sarkar et al. (2009), p. 45, pl. 10, fig. 12.

Trifarina carinata: Nisha (2002), p. 147, pl. 4, figs. 15-16.

Triloculina tricarinata: Sarkar et al. (2009), p. 45, pl. 10, fig. 17.

Trochammina rugosa: Parr (1950), p. 233-392, pls. 3-5, figs. 1-8.

Trochammina sphaeriloculum: Haplophragmoides sphaeriloculum para Cushman (1920), p. 44, pl. 8, fig. 3.

Uvigerina ampullacea: Uvigerina asperula var. ampullacea para Xia and Fan (1996), p. 139, pl. 1, figs. 14-15.

Uvigerina excellens: Matoba and Yamaguchi (1982), p. 1054, pl. 2, fig. 9.

Uvigerina hispida: McDougall (1985), p. 416, pl. 4, fig. 5; Bornmalm (1997), p. 50, fig. 19h.

Uvigerina hispidocostata: Akimoto (1994), p. 289, pl. 2, fig. 18-20.

Uvigerina incilis: Gualancañay (1983), p. 609, pl. 7, fig. 11.

Uvigerina mantaensis: Bornmalm (1997), p. 50, fig. 19k.

Uvigerina peregrina: Ohkushi et al. (2000), p. 140, pl. 2, fig. 4.

Uvigerina peregrina curticosa: Matoba and Yamaguchi (1982), p. 1054, pl. 2, figs. 10-12.

Uvigerina proboscidea: Akimoto (1990), pl. 22, fig. 12; Fiorini (2015), p. 125, pl. 2, fig. 8.

Uvigerina senticosa: Kaiho (1989), p. 306, pl. 4, fig. 12.

Uvigerina vadescens: Betancur y Martínez (2003), p. 120, pl. 1, fig. 9.

Valvulineria humilis: Bornmalm (1997), p. 54, figs. 20h-i.

Valvulineria minuta: Sarkar et al. (2009), p. 46, pl. 11, fig. 10. 